\title{
Quasilinear boundary value problem with impulses: variational approach to resonance problem
}

\author{
Pavel Drábek ${ }^{1,2}$ and Martina Langerová2*
}

We dedicate this paper to Professor Ivan Kiguradze for his merits in the theory of differential equations.

${ }^{*}$ Correspondence:

mlanger@ntis.zcu.cz

${ }^{2}$ NTIS, University of West Bohemia,

Univerzitní 22, Plzeň, 306 14, Czech Republic

Full list of author information is available at the end of the article

\begin{abstract}
This paper deals with the resonance problem for the one-dimensional $p$-Laplacian with homogeneous Dirichlet boundary conditions and with nonlinear impulses in the derivative of the solution at prescribed points. The sufficient condition of Landesman-Lazer type is presented and the existence of at least one solution is proved. The proof is variational and relies on the linking theorem.

MSC: Primary 34A37; 34B37; secondary 34F15; 49K35
\end{abstract}

Keywords: quasilinear impulsive differential equations; Landesman-Lazer condition; variational methods; critical point theory; linking theorem

\section{Introduction}

Let $p>1$ be a real number. We consider the homogeneous Dirichlet boundary value problem for one-dimensional $p$-Laplacian

$$
\begin{aligned}
& -\left(\left|u^{\prime}(x)\right|^{p-2} u^{\prime}(x)\right)^{\prime}-\lambda|u(x)|^{p-2} u(x)=f(x) \quad \text { for a.e. } x \in(0,1), \\
& u(0)=u(1)=0
\end{aligned}
$$

where $\lambda \in \mathbb{R}$ is a spectral parameter and $f \in L^{p^{\prime}}(0,1), \frac{1}{p}+\frac{1}{p^{\prime}}=1$, is a given right-hand side. Let $0=t_{0}<t_{1}<\cdots<t_{r}<t_{r+1}=1$ be given points and let $I_{j}: \mathbb{R} \rightarrow \mathbb{R}, j=1,2, \ldots, r$, be given continuous functions. We are interested in the solutions of (1) satisfying the impulse conditions in the derivative

$$
\Delta_{p} u^{\prime}\left(t_{j}\right):=\left|u^{\prime}\left(t_{j}^{+}\right)\right|^{p-2} u^{\prime}\left(t_{j}^{+}\right)-\left|u^{\prime}\left(t_{j}^{-}\right)\right|^{p-2} u^{\prime}\left(t_{j}^{-}\right)=I_{j}\left(u\left(t_{j}\right)\right), \quad j=1,2, \ldots, r .
$$

For the sake of brevity, in further text we use the following notation:

$$
\varphi(s):=|s|^{p-2} s, \quad s \neq 0 ; \quad \varphi(0):=0 .
$$

For $p=2$ this problem is considered in [1] where the necessary and sufficient condition for the existence of a solution of (1) and (2) is given. In fact, in the so-called resonance case, we introduce necessary and sufficient conditions of Landesman-Lazer type in terms

C2014 Drábek and Langerová; licensee Springer. This is an Open Access article distributed under the terms of the Creative Commons Attribution License (http://creativecommons.org/licenses/by/2.0), which permits unrestricted use, distribution, and reproduction in any medium, provided the original work is properly cited. 
of the impulse functions $I_{j}, j=1,2, \ldots, r$, and the right-hand side $f$. They generalize the Fredholm alternative for linear problem (1) with $p=2$.

In this paper we focus on a quasilinear equation with $p \neq 2$ and look just for sufficient conditions. We point out that there are principal differences between the linear case $(p=2)$ and the nonlinear case $(p \neq 2)$. In the linear case, we could benefit from the Hilbert structure of an abstract formulation of the problem. It could be treated using the topological degree as a nonlinear compact perturbation of a linear operator. However, in the nonlinear case, completely different approach must be chosen in the resonance case. Our variational proof relies on the linking theorem (see [2]), but we have to work in a Banach space since the Hilbert structure is not suitable for the case $p \neq 2$.

It is known that the eigenvalues of

$$
\begin{aligned}
& -\left(\varphi\left(u^{\prime}(x)\right)\right)^{\prime}-\lambda \varphi(u(x))=0, \\
& u(0)=u(1)=0
\end{aligned}
$$

are simple and form an unbounded increasing sequence $\left\{\lambda_{n}\right\}$ whose eigenspaces are spanned by functions $\left\{\phi_{n}(x)\right\} \subset W_{0}^{1, p}(0,1) \cap C^{1}[0,1]$ such that $\phi_{n}$ has $n-1$ evenly spaced zeros in $(0,1),\left\|\phi_{n}\right\|_{L^{p}(0,1)}=1$, and $\phi_{n}^{\prime}(0)>0$. The reader is invited to see [3, p.388], [4, p.780] or [5, pp.272-275] for further details. See also Example 1 below for more explicit form of $\lambda_{n}$ and $\phi_{n}$.

Let $\lambda \neq \lambda_{n}, n=1,2, \ldots$, in (1). This is the nonresonance case. Then, for any $f \in L^{p^{\prime}}(0,1)$, there exists at least one solution of (1). In the case $p=2$, this solution is unique. In the case $p \neq 2$, the uniqueness holds if $\lambda \leq 0$, but it may fail for certain right-hand sides $f \in L^{p^{\prime}}(0,1)$ if $\lambda>0$. See, e.g., [6] (for $2<p<\infty$ ) and [7] (for $1<p<2$ ).

The same argument as that used for $p=2$ in [1, Section 3] for the nonresonance case yields the following existence result for the quasilinear impulsive problem (1), (2).

Theorem 1 (Nonresonance case) Let $\lambda \neq \lambda_{n}, n=1,2, \ldots, I_{j}: \mathbb{R} \rightarrow \mathbb{R}, j=1,2, \ldots, r$, be continuous functions which are $(p-1)$-subhomogeneous at $\pm \infty$, that is,

$$
\lim _{|s| \rightarrow \infty} \frac{I_{j}(s)}{|s|^{p-2} s}=0
$$

Then (1), (2) has a solution for arbitrary $f \in L^{p^{\prime}}(0,1)$.

Variational approach to impulsive differential equations of the type (1), (2) with $p=2$ was used, e.g., in paper [8]. The authors apply the mountain pass theorem to prove the existence of a solution for $\lambda<\lambda_{1}$. Our Theorem 1 thus generalizes [8, Theorem 5.2] in two directions. Firstly, it allows also $\lambda>\lambda_{1}\left(\lambda \neq \lambda_{n}, n=2,3, \ldots\right)$ and, secondly, it deals with quasilinear equations $(p \neq 2)$, too.

Let $\lambda=\lambda_{n}$ for some $n \in \mathbb{N}$. This is the resonance case. Contrary to the linear case $(p=2)$, there is no Fredholm alternative for (1) in the nonlinear case $(p \neq 2)$. If $\lambda=\lambda_{1}$, then

$$
f \in \phi_{1}^{\perp}:=\left\{h \in L^{\infty}(0,1): \int_{0}^{1} h(x) \phi_{1}(x) \mathrm{d} x=0\right\}
$$

is the sufficient condition for solvability of (1), but it is not necessary if $p \neq 2$. Moreover, if $f \notin \phi_{1}^{\perp}$ but $f$ is 'close enough' to $\phi_{1}^{\perp}$, problem (1) has at least two distinct solutions. The 
reader is referred to [3] or [9] for more details. It appears that the situation is even more complicated for $\lambda=\lambda_{n}, n \geq 2$ (see, e.g., [10]).

In the presence of nonlinear impulses which have certain asymptotic properties (to be made precise below), we show that the fact $f \in \phi_{n}^{\perp}$ might still be the sufficient condition for the existence of a solution to (1) (with $\lambda=\lambda_{n}$ ) and (2). For this purpose we need some notation. Let $0<x_{1}<x_{2}<\cdots<x_{n-1}<1$ denote evenly spaced zeros of $\phi_{n}$, let $\mathcal{I}_{+}=\left(0, x_{1}\right) \cup$ $\left(x_{2}, x_{3}\right) \cup \cdots$ and $\mathcal{I}_{-}=\left(x_{1}, x_{2}\right) \cup\left(x_{3}, x_{4}\right) \cup \cdots$ denote the union of intervals where $\phi_{n}>$ 0 or $\phi_{n}<0$, respectively. We arrange $t_{j}, j=1,2, \ldots, r$, into three sequences: $0<\tau_{1}<\tau_{2}<$ $\cdots<\tau_{r_{+}}<1, \tau_{i} \in \mathcal{I}_{+}, i=1,2, \ldots, r_{+} ; 0<\sigma_{1}<\sigma_{2}<\cdots<\sigma_{r_{-}}<1, \sigma_{j} \in \mathcal{I}_{-}, j=1,2, \ldots, r_{-} ; \xi_{k} \in$ $\left\{x_{1}, x_{2}, \ldots, x_{n-1}\right\}, k=1,2, \ldots, r_{0}$. Obviously, we have $r_{+}+r_{-}+r_{0}=r$ and $r_{0} \leq n-1$. Assume that $r_{+}+r_{-}>0$, i.e., $r_{0}<n-1$. The impulse condition (2) can be written in an equivalent form

$$
\begin{aligned}
& \Delta_{p} u^{\prime}\left(\tau_{i}\right)=I_{i}^{\tau}\left(u\left(\tau_{i}\right)\right), \quad i=1,2, \ldots, r_{+}, \\
& \Delta_{p} u^{\prime}\left(\sigma_{j}\right)=I_{j}^{\sigma}\left(u\left(\sigma_{j}\right)\right), \quad j=1,2, \ldots, r_{-}, \\
& \Delta_{p} u^{\prime}\left(\xi_{k}\right)=I_{k}^{\xi}\left(u\left(\xi_{k}\right)\right), \quad k=1,2, \ldots, r_{0} .
\end{aligned}
$$

We assume that $I_{i}^{\tau}, I_{j}^{\sigma}, I_{k}^{\xi}: \mathbb{R} \rightarrow \mathbb{R}, i=1,2, \ldots, r_{+} ; j=1,2, \ldots, r_{-} ; k=1,2, \ldots, r_{0}$, are contin-

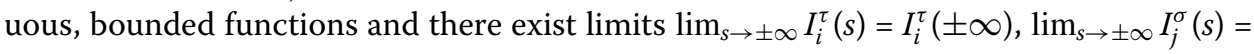
$I_{j}^{\sigma}( \pm \infty)$. We consider the following Landesman-Lazer type conditions: either

$$
\begin{aligned}
\sum_{i=1}^{r_{+}} I_{i}^{\tau}(-\infty) \phi_{n}\left(\tau_{i}\right)+\sum_{j=1}^{r_{-}} I_{j}^{\sigma}(+\infty) \phi_{n}\left(\sigma_{j}\right) & <\int_{0}^{1} f(x) \phi_{n}(x) \mathrm{d} x \\
& <\sum_{i=1}^{r_{+}} I_{i}^{\tau}(+\infty) \phi_{n}\left(\tau_{i}\right)+\sum_{j=1}^{r_{-}} I_{j}^{\sigma}(-\infty) \phi_{n}\left(\sigma_{j}\right)
\end{aligned}
$$

or

$$
\begin{aligned}
\sum_{i=1}^{r_{+}} I_{i}^{\tau}(+\infty) \phi_{n}\left(\tau_{i}\right)+\sum_{j=1}^{r_{-}} I_{j}^{\sigma}(-\infty) \phi_{n}\left(\sigma_{j}\right) & <\int_{0}^{1} f(x) \phi_{n}(x) \mathrm{d} x \\
& <\sum_{i=1}^{r_{+}} I_{i}^{\tau}(-\infty) \phi_{n}\left(\tau_{i}\right)+\sum_{j=1}^{r_{-}} I_{j}^{\sigma}(+\infty) \phi_{n}\left(\sigma_{j}\right) .
\end{aligned}
$$

Our main result is the following.

Theorem 2 (Resonance case) Let $\lambda=\lambda_{n}$ for some $n \in \mathbb{N}$ in (1). Let the nonlinear bounded impulse functions $I_{j}: \mathbb{R} \rightarrow \mathbb{R}, j=1,2, \ldots, r$, and the right-hand side $f \in L^{p^{\prime}}(0,1)$ satisfy either (5) or (6). Then (1), (2) has a solution.

The result from Theorem 2 is illustrated in the following special example.

Example 1 It follows from the first integral associated with the equation in (3) that the eigenvalues and the eigenfunctions of (3) have the form

$$
\lambda_{n}=(p-1)\left(n \pi_{p}\right)^{p}, \quad \phi_{n}(x)=\frac{\sin _{p}\left(n \pi_{p} x\right)}{\left\|\sin _{p}\left(n \pi_{p} x\right)\right\|_{L^{p}(0,1)}},
$$


where $\pi_{p}=\frac{2 \pi}{p \sin \frac{\pi}{p}}$ and $x=\int_{0}^{\sin _{p} x} \frac{\mathrm{d} s}{\left(1-s^{p}\right)^{\frac{1}{p}}}, x \in\left[0, \frac{\pi_{p}}{2}\right], \sin _{p} x=\sin _{p}\left(\pi_{p}-x\right), x \in\left[\frac{\pi_{p}}{2}, \pi_{p}\right], \sin _{p} x=$ $-\sin _{p}\left(2 \pi_{p}-x\right), x \in\left[\pi_{p}, 2 \pi_{p}\right]$, see [3, p.388]. Let us consider $\lambda=\lambda_{2}$ in (1) and $t_{1}=\frac{\pi_{p}}{4}, t_{2}=$ $\frac{3 \pi_{p}}{4}, I_{j}(s)=\arctan s, s \in \mathbb{R}, j=1,2$, in (2). Since $\sin \frac{\pi_{p}}{2}=\frac{1}{p-1}, \sin \frac{3 \pi_{p}}{2}=-\frac{1}{p-1}$, condition (6) reads as follows:

$$
-\frac{\pi}{p-1}<\int_{0}^{1} f(x) \sin _{p} 2 \pi_{p} x \mathrm{~d} x<\frac{\pi}{p-1} .
$$

\section{Functional framework}

We say that $u$ is the classical solution of (1), (2) if the following conditions are fulfilled:

- $u \in C[0,1], u \in C^{1}\left(t_{j}, t_{j+1}\right), \varphi\left(u^{\prime}(\cdot)\right)$ is absolutely continuous in $\left(t_{j}, t_{j+1}\right), j=0,1, \ldots, r$;

- the equation in $(1)$ holds a.e. in $(0,1)$ and $u(0)=u(1)=0$;

- one-sided limits $u^{\prime}\left(t_{j}^{+}\right), u^{\prime}\left(t_{j}^{-}\right)$exist finite and (2) holds.

We say that $u \in W_{0}^{1, p}(0,1)$ is a weak solution of $(1),(2)$ if the integral identity

$$
\int_{0}^{1} \varphi\left(u^{\prime}(x)\right) v^{\prime}(x) \mathrm{d} x-\lambda \int_{0}^{1} \varphi(u(x)) v(x) \mathrm{d} x+\sum_{j=1}^{r} I_{j}\left(u\left(t_{j}\right)\right) v\left(t_{j}\right)=\int_{0}^{1} f(x) v(x) \mathrm{d} x
$$

holds for any function $v \in W_{0}^{1, p}(0,1)$.

Integration by parts and the fundamental lemma in calculus of variations (see [11, Lemma 7.1.9]) yields that every weak solution of (1), (2) is also a classical solution and vice versa. Indeed, let $u$ be a weak solution of (1), (2), $v \in \mathcal{D}\left(t_{j}, t_{j+1}\right)$ (the space of smooth functions with a compact support in $\left.\left(t_{j}, t_{j+1}\right), j=0,1, \ldots, r\right), v \equiv 0$ elsewhere in $(0,1)$, then

$$
\int_{t_{j}}^{t_{j+1}}\left(\varphi\left(u^{\prime}(x)\right)+\int_{0}^{x}[\lambda \varphi(u(\tau))+f(\tau)] \mathrm{d} \tau\right) v^{\prime}(x) \mathrm{d} x=0 .
$$

Since $v$ is arbitrary, we have $\varphi\left(u^{\prime}(x)\right)+\int_{0}^{x}[\lambda \varphi(u(\tau))+f(\tau)] \mathrm{d} \tau=0$ for a.e. $x \in\left(t_{j}, t_{j+1}\right)$. Then $\varphi\left(u^{\prime}(\cdot)\right)$ is absolutely continuous in $\left(t_{j}, t_{j+1}\right)$ and

$$
-\left(\varphi\left(u^{\prime}(x)\right)\right)^{\prime}-\lambda \varphi(u(x))=f(x)
$$

for a.e. $x \in\left(t_{j}, t_{j+1}\right), j=0,1, \ldots, r$. Taking now $v \in W_{0}^{1, p}(0,1)$ arbitrary, integrating by parts in the first integral in (7) and using (8), we get

$$
\sum_{j=1}^{r}\left[\varphi\left(u^{\prime}\left(t_{j}^{+}\right)\right)-\varphi\left(u^{\prime}\left(t_{j}^{-}\right)\right)\right] v\left(t_{j}\right)=\sum_{j=1}^{r} I_{j}\left(u\left(t_{j}\right)\right) v\left(t_{j}\right)
$$

and hence also (2) follows. Similarly, we show that every classical solution is a weak solution at the same time.

Let $X:=W_{0}^{1, p}(0,1)$ with the norm $\|u\|=\left(\int_{0}^{1}\left|u^{\prime}(x)\right|^{p} \mathrm{~d} x\right)^{\frac{1}{p}}, X^{*}$ be the dual of $X$ and $\langle\cdot, \cdot\rangle$ be the duality pairing between $X^{*}$ and $X$. For $u \in X$, we set

$$
\begin{array}{ll}
A(u):=\frac{1}{p} \int_{0}^{1}\left|u^{\prime}(x)\right|^{p} \mathrm{~d} x, & B(u):=\frac{1}{p} \int_{0}^{1}|u(x)|^{p} \mathrm{~d} x, \\
F(u)=\int_{0}^{1} f(x) u(x) \mathrm{d} x, & J(u):=\sum_{j=1}^{r} \int_{0}^{u\left(t_{j}\right)} I_{j}(s) \mathrm{d} s .
\end{array}
$$


Then, for $u, v \in X$, we have

$$
\begin{aligned}
& \left\langle A^{\prime}(u), v\right\rangle=\int_{0}^{1} \varphi\left(u^{\prime}(x)\right) v^{\prime}(x) \mathrm{d} x, \quad\left\langle B^{\prime}(u), v\right\rangle=\int_{0}^{1} \varphi(u(x)) v(x) \mathrm{d} x, \\
& \left\langle F^{\prime}, v\right\rangle=\int_{0}^{1} f(x) v(x) \mathrm{d} x, \quad\left\langle J^{\prime}(u), v\right\rangle=\sum_{j=1}^{r} I_{j}\left(u\left(t_{j}\right)\right) v\left(t_{j}\right) .
\end{aligned}
$$

Lemma 1 The operators $A^{\prime}, B^{\prime}, J^{\prime}: X \rightarrow X^{*}$ have the following properties:

(A) $A^{\prime}$ is $(p-1)$-homogeneous, odd, continuously invertible, and $\left\|A^{\prime}(u)\right\|_{*}=\|u\|^{p-1}$ for any $u \in X$.

(B) $B^{\prime}$ is $(p-1)$-homogeneous, odd and compact.

(J) $J^{\prime}$ is bounded and compact.

By the linearity of $F: X \rightarrow \mathbb{R}, F^{\prime} \in X^{*}$ is a fixed element.

Proof See [12, Lemma 10.3, p.120].

With this notation in hands we can look for (classical) solutions of (1), (2) either as for solutions $u \in X$ of the operator equation

$$
A^{\prime}(u)-\lambda B^{\prime}(u)+J^{\prime}(u)=F^{\prime}
$$

or, alternatively, as for critical points of the functional $\mathcal{F}: X \rightarrow \mathbb{R}$,

$$
\mathcal{F}(u):=A(u)-\lambda B(u)+J(u)-F(u)
$$

As mentioned already above, in the nonresonance case $\left(\lambda \neq \lambda_{n}, n \in \mathbb{N}\right)$, we can use the Leray-Schauder degree argument and prove the existence of a solution of the equation (9) exactly as in [1, proof of Thm. 1]. Note that the $(p-1)$-subhomogeneous condition on $I_{j}$ is used here instead of the sublinear condition imposed on $I_{j}$ in [1] and the proof of Theorem 1 follows the same lines. For this reason we skip it and concentrate on the resonance case $\left(\lambda=\lambda_{n}\right.$ for some $\left.n \in \mathbb{N}\right)$ in the next section.

\section{Resonance problem, variational approach}

We use the following definition of linked sets and the linking theorem (cf. [13]).

Definition 1 Let $\mathcal{E}$ be a closed subset of $X$ and let $Q$ be a submanifold of $X$ with relative boundary $\partial Q$. We say that $\mathcal{E}$ and $\partial Q$ link if

(i) $\mathcal{E} \cap \partial Q=\varnothing$ and

(ii) for any continuous map $h: X \rightarrow X$ such that $h \mid \partial Q=\mathrm{id}$, there holds $h(Q) \cap \mathcal{E} \neq \emptyset$.

(See [14, Def. 8.1, p.116].)

Theorem 3 (Linking theorem) Suppose that $\mathcal{F} \in C^{1}(X)$ satisfies the Palais-Smale condition. Consider a closed subset $\mathcal{E} \subset X$ and a submanifold $Q \subset X$ with relative boundary $\partial Q$, and let $\Gamma:=\left\{h \in C^{0}(X, X): h \mid \partial Q=\mathrm{id}\right\}$. Suppose that $\mathcal{E}$ and $\partial Q$ link in the sense of Defini- 
tion 1 , and

$$
\inf _{u \in \mathcal{E}} \mathcal{F}(u)>\sup _{u \in \partial Q} \mathcal{F}(u)
$$

Then $\beta=\inf _{h \in \Gamma} \sup _{u \in Q} \mathcal{F}(h(u))$ is a critical value of $\mathcal{F}$.

(See [14, Thm. 8.4, p.118].)

The purpose of the following series of lemmas is to show that the hypotheses of Theorem 3 are satisfied provided that either (5) or (6) holds. From now on we assume that $\lambda=\lambda_{n}$ (for some $\left.n \in \mathbb{N}\right)$ in (1).

Lemma 2 If either (5) or (6) is satisfied, then $\mathcal{F}$ satisfies the Palais-Smale condition.

Proof Suppose that $\left\{u_{k}\right\} \in X$ such that $\left|\mathcal{F}\left(u_{k}\right)\right| \leq c$ and $\mathcal{F}^{\prime}\left(u_{k}\right) \rightarrow 0$ in $X^{*}$. We must show that $\left\{u_{k}\right\}$ has a subsequence that converges in $X$. We prove first that $\left\{u_{k}\right\}$ is a bounded sequence. We proceed via contradiction and suppose that $\left\|u_{k}\right\| \rightarrow \infty$ and consider $v_{k}:=$ $\frac{u_{k}}{\left\|u_{k}\right\|}$. Without loss of generality, we can assume that there is $v_{0} \in X$ such that $v_{k} \rightarrow v_{0}$ (weakly) in $X$ ( $X$ is a reflexive Banach space). Since

$$
0 \leftarrow \mathcal{F}^{\prime}\left(u_{k}\right)=A^{\prime}\left(u_{k}\right)-\lambda_{n} B^{\prime}\left(u_{k}\right)+J^{\prime}\left(u_{k}\right)-F^{\prime}
$$

dividing through by $\left\|u_{k}\right\|^{p-1}$, we have

$$
A^{\prime}\left(v_{k}\right)-\lambda_{n} B^{\prime}\left(v_{k}\right)+\frac{J^{\prime}\left(u_{k}\right)}{\left\|u_{k}\right\|^{p-1}}-\frac{F^{\prime}}{\left\|u_{k}\right\|^{p-1}} \rightarrow 0 .
$$

By the boundedness of $J^{\prime}$ we know that $\frac{J^{\prime}\left(u_{k}\right)}{\left\|u_{k}\right\|^{p-1}} \rightarrow 0$. We also have $\frac{F^{\prime}}{\left\|u_{k}\right\|^{p-1}} \rightarrow 0$. By the compactness of $B^{\prime}$ we get $B^{\prime}\left(v_{k}\right) \rightarrow B^{\prime}\left(v_{0}\right)$ in $X^{*}$. Thus $v_{k} \rightarrow v_{0}=\left(A^{\prime}\right)^{-1}\left(\lambda_{n} B^{\prime}\left(v_{0}\right)\right)$ in $X$ by Lemma 1 (A). It follows that $v_{0}= \pm \frac{1}{\lambda_{n}^{\frac{1}{p}}} \phi_{n}$.

We assume $v_{0}=\frac{1}{\lambda_{n}^{\frac{1}{p}}} \phi_{n}$ and remark that a similar argument follows if $v_{0}=-\frac{1}{\lambda_{n}^{\frac{1}{p}}} \phi_{n}$. Next we estimate

$$
p \mathcal{F}\left(u_{k}\right)-\left\langle\mathcal{F}^{\prime}\left(u_{k}\right), u_{k}\right\rangle=p J\left(u_{k}\right)-\left\langle J^{\prime}\left(u_{k}\right), u_{k}\right\rangle+(1-p) \int_{0}^{1} f(x) u_{k}(x) \mathrm{d} x
$$

Our assumption $\left|\mathcal{F}\left(u_{k}\right)\right| \leq c$ yields

$$
-c p \leq p \mathcal{F}\left(u_{k}\right) \leq c p
$$

and the Cauchy-Schwarz inequality implies

$$
-\left\|u_{k}\right\|\left\|\mathcal{F}^{\prime}\left(u_{k}\right)\right\|_{*} \leq-\left\langle\mathcal{F}^{\prime}\left(u_{k}\right), u_{k}\right\rangle \leq\left\|u_{k}\right\|\left\|\mathcal{F}^{\prime}\left(u_{k}\right)\right\|_{*^{\prime}}
$$

where $\|\cdot\|_{*}$ denotes the norm in $X^{*}$. It follows from (11)-(13) that

$$
\begin{aligned}
-c p-\left\|u_{k}\right\|\left\|\mathcal{F}^{\prime}\left(u_{k}\right)\right\|_{*} & \leq p J\left(u_{k}\right)-\left\langle J^{\prime}\left(u_{k}\right), u_{k}\right\rangle+(1-p) \int_{0}^{1} f(x) u_{k}(x) \mathrm{d} x \\
& \leq c p+\left\|u_{k}\right\|\left\|\mathcal{F}^{\prime}\left(u_{k}\right)\right\|_{*^{*}}
\end{aligned}
$$


Dividing through by $\left\|u_{k}\right\|$ and writing $\frac{\int_{0}^{u_{k}\left(t_{j}\right)} I_{j}(s) \mathrm{d} s}{\left\|u_{k}\right\|}=\hat{I}_{j}\left(u_{k}\left(t_{j}\right)\right) v_{k}\left(t_{j}\right)$, where

$$
\hat{I}_{j}(\sigma):= \begin{cases}\frac{\int_{0}^{\sigma} I_{j}(s) \mathrm{d} s}{\sigma} & \text { for } \sigma \neq 0 \\ 0 & \text { for } \sigma=0\end{cases}
$$

$j=0,1, \ldots, r$, we get

$$
\begin{aligned}
& \left|p \sum_{j=1}^{r} \hat{I}_{j}\left(u_{k}\left(t_{j}\right)\right) v_{k}\left(t_{j}\right)-\sum_{j=1}^{r} I_{j}\left(u_{k}\left(t_{j}\right)\right) v_{k}\left(t_{j}\right)+(1-p) \int_{0}^{1} f(x) v_{k}(x) \mathrm{d} x\right| \\
& \quad \leq \frac{c p}{\left\|u_{k}\right\|}+\left\|\mathcal{F}^{\prime}\left(u_{k}\right)\right\|_{*} \rightarrow 0 .
\end{aligned}
$$

Since $\int_{0}^{1} f(x) v_{k}(x) \mathrm{d} x \rightarrow \frac{1}{\lambda_{n}^{\frac{1}{p}}} \int_{0}^{1} f(x) \phi_{n}(x) \mathrm{d} x$ as $k \rightarrow \infty$, we obtain from (14):

$$
\lim _{k \rightarrow \infty} \sum_{j=1}^{r}\left(p \hat{I}_{j}\left(u_{k}\left(t_{j}\right)\right)-I_{j}\left(u_{k}\left(t_{j}\right)\right)\right) \nu_{k}\left(t_{j}\right)=\frac{p-1}{\lambda_{n}^{\frac{1}{p}}} \int_{0}^{1} f(x) \phi_{n}(x) \mathrm{d} x .
$$

Recall that $X$ embeds compactly in $C[0,1]$, so, without loss of generality, we assume that $v_{k}\left(t_{j}\right) \rightarrow \frac{1}{\lambda_{n}^{\frac{1}{p}}} \phi_{n}\left(t_{j}\right), j=0,1, \ldots, r$, as $k \rightarrow \infty$. Hence, $u_{k}\left(t_{j}\right) \rightarrow \pm \infty$ for $t_{j} \in \mathcal{I}_{ \pm}$, which implies $I_{j}\left(u_{k}\left(t_{j}\right)\right) \rightarrow I_{j}( \pm \infty)$ as well as $\hat{I}_{j}\left(u_{k}\left(t_{j}\right)\right) \rightarrow I_{j}( \pm \infty)$ as $k \rightarrow \infty$ by an application of the l'Hospital rule to $\frac{\int_{0}^{\sigma} I_{j}(s) \mathrm{d} s}{\sigma}$. Notice that by the boundedness of $I_{j}$ we have

$$
\left(p \hat{I}_{j}\left(u_{k}\left(t_{j}\right)\right)-I_{j}\left(u_{k}\left(t_{j}\right)\right)\right) v_{k}\left(t_{j}\right) \rightarrow 0 \quad \text { as } k \rightarrow \infty
$$

if $t_{j}$ is a zero point of $\phi_{n}$ for some $j \in\{1,2, \ldots, r\}$. Thus, passing to the limit in (15) as $k \rightarrow \infty$, we get

$$
\sum_{i=1}^{r_{+}} I_{i}^{\tau}(+\infty) \phi_{n}\left(\tau_{i}\right)+\sum_{j=1}^{r_{-}} I_{j}^{\sigma}(-\infty) \phi_{n}\left(\sigma_{j}\right)=\int_{0}^{1} f(x) \phi_{n}(x) \mathrm{d} x,
$$

which contradicts (5) or (6). Hence $\left\{u_{k}\right\}$ is bounded.

By compactness there is a subsequence such that $B^{\prime}\left(u_{k}\right)$ and $J^{\prime}\left(u_{k}\right)$ converge in $X^{*}$ (see Lemma $1(\mathrm{~B}),(\mathrm{J}))$. Since $\mathcal{F}^{\prime}\left(u_{k}\right) \rightarrow 0$ by our assumption, we also have that $A^{\prime}\left(u_{k}\right)$ converges in $X^{*}$. Finally, $u_{k}=\left(A^{\prime}\right)^{-1}\left(A^{\prime}\left(u_{k}\right)\right)$ converges in $X$ by Lemma $1(\mathrm{~A})$. The proof is finished.

With the Palais-Smale condition in hands, we can turn our attention to the geometry of the functional $\mathcal{F}$. To this end we have to find suitable sets which link in the sense of Definition 1. Actually, we use the sets constructed in [13] and explain that they fit with the hypotheses of Theorem 3 if either (5) or (6) is satisfied.

Consider the even functional

$$
E(u):=\frac{A(u)}{B(u)} \quad \text { for } u \in X \backslash\{0\}
$$


and the manifold

$$
\mathcal{S}:=\left\{u \in W_{0}^{1, p}(0,1): B(u)=1\right\} .
$$

For any $n \in \mathbb{N}$, let $\mathcal{F}_{n}:=\left\{\mathcal{A} \subset \mathcal{S}: \exists\right.$ continuous odd surjection $\left.h: \mathcal{S}^{n-1} \rightarrow \mathcal{A}\right\}$, where $\mathcal{S}^{n-1}$ represents the unit sphere in $\mathbb{R}^{n}$. Next we define

$$
\lambda_{n}:=\inf _{\mathcal{A} \in \mathcal{F}_{n}} \sup _{u \in \mathcal{A}} E(u), \quad n \in \mathbb{N} .
$$

It is proved in $[15$, Section 3$]$ that $\left\{\lambda_{n}\right\}$ is a sequence of eigenvalues of homogeneous problem (3). It then follows from the results in [16] that this sequence exhausts the set of all eigenvalues of (3) with the properties described in Section 1.

Now consider the functions $\phi_{n, i}=\chi_{\left[\frac{i-1}{n}, \frac{i}{n}\right]} \phi_{n}$ for $i=1,2, \ldots, n$, where $\chi_{\left[\frac{i-1}{n}, \frac{i}{n}\right]}$ is a characteristic function of the interval $\left[\frac{i-1}{n}, \frac{i}{n}\right]$, and let

$$
\Lambda_{n}:=\left\{\alpha_{1} \phi_{n, 1}+\cdots+\alpha_{n} \phi_{n, n}: \alpha_{i} \in \mathbb{R} \text { and }\left|\alpha_{1}\right|^{p} B\left(\phi_{n, 1}\right)+\cdots+\left|\alpha_{n}\right|^{p} B\left(\phi_{n, n}\right)=1\right\} \text {. }
$$

Observe that $\Lambda_{n}$ is symmetric and is homeomorphic to the unit sphere in $\mathbb{R}^{n}$. Moreover, for $u \in \Lambda_{n}$, we have

$$
\begin{aligned}
B(u) & =B\left(\alpha_{1} \phi_{n, 1}+\cdots+\alpha_{n} \phi_{n, n}\right)=B\left(\alpha_{1} \phi_{n, 1}\right)+\cdots+B\left(\alpha_{n} \phi_{n, n}\right) \\
& =\left|\alpha_{1}\right|^{p} B\left(\phi_{n, 1}\right)+\cdots+\left|\alpha_{n}\right|^{p} B\left(\phi_{n, n}\right)=1 .
\end{aligned}
$$

Notice that the second equality holds thanks to the fact

$$
\left\{x: \phi_{n, i}(x) \neq 0\right\} \cap\left\{x: \phi_{n, j}(x) \neq 0\right\}=\emptyset
$$

for $i \neq j, i, j=1,2, \ldots, n$, while the third one follows from the $p$-homogeneity of $B$. Thus $\Lambda_{n} \subset \mathcal{S}$ and so $\Lambda_{n} \in \mathcal{F}_{n}$. A similar computation then shows that $E(u)=A(u)=\lambda_{n}$ for all $u \in \Lambda_{n}$. For a given $T>0$, we let

$$
Q_{n, T}:=\left\{s u: 0 \leq s \leq T, u \in \Lambda_{n}\right\}
$$

Then $Q_{n, T}$ is homeomorphic to the closed unit ball in $\mathbb{R}^{n}$. For a given $c \in \mathbb{R}$, we denote by

$$
\mathcal{E}_{c}:=\{u \in X: A(u) \geq c B(u)\}=\{u \in X \backslash\{0\}: E(u) \geq c\} \cup\{0\}
$$

a super-level set, and

$$
\mathcal{K}_{c}:=\left\{u \in X \backslash\{0\}: E(u)=c, E^{\prime}(u)=0\right\} .
$$

The existence of a pseudo-gradient vector field with the following properties is proved in [13, Lemma 6] (cf. [14, pp.77-79] and [2, p.55]).

Lemma 3 For $\varepsilon<\min \left\{\lambda_{n+1}-\lambda_{n}, \lambda_{n}-\lambda_{n-1}\right\}$, there is $\tilde{\varepsilon} \in(0, \varepsilon)$ and a one-parameter family of homeomorphisms $\eta:[-1,1] \times \mathcal{S} \rightarrow \mathcal{S}$ such that 
(i) $\eta(t, u)=u$ if $E(u) \in\left(-\infty, \lambda_{n}-\varepsilon\right] \cup\left[\lambda_{n}+\varepsilon, \infty\right)$ or if $u \in \mathcal{K}_{\lambda_{n}}$;

(ii) $E(\eta(t, u))$ is strictly decreasing in $t$ if $E(u) \in\left(\lambda_{n}-\tilde{\varepsilon}_{n}, \lambda_{n}+\tilde{\varepsilon}_{n}\right)$ and $u \notin \mathcal{K}_{\lambda_{n}}$;

(iii) $\eta(t,-u)=-\eta(t, u)$;

(iv) $\eta(0, \cdot)=\mathrm{id}$

An important fact is that the flow $\eta$ 'lowers' $Q_{n, T}$ and 'raises' $\mathcal{E}_{\lambda_{n}}$ if we modify them as follows:

$$
\tilde{\mathcal{E}}_{\lambda_{n}}:=\left\{s u: s \in \mathbb{R}, u \in \eta\left(-1, \mathcal{E}_{\lambda_{n}} \cap \mathcal{S}\right)\right\}
$$

and

$$
\tilde{Q}_{n, T}:=\left\{s u: 0 \leq s \leq T, u \in \eta\left(1, \Lambda_{n}\right)\right\} .
$$

Then, by Lemma 3 and the definition of $\mathcal{E}_{\lambda_{n}}$, we have

$$
A(u)-\lambda_{n} B(u) \geq 0
$$

for $u \in \tilde{\mathcal{E}}_{\lambda_{n}}$ with equality if and only if $u=c \phi_{n}$ for some $c \in \mathbb{R}$. Similarly,

$$
A(u)-\lambda_{n} B(u) \leq 0
$$

for $u \in \tilde{Q}_{n, T}$ with equality if and only if $u=c \phi_{n}$ for some $c \in \mathbb{R}$.

It is proved in [13, Lemma 7] that the couple $\mathcal{E}:=\mathcal{E}_{\lambda_{n+1}}$ and $Q:=\tilde{Q}_{n, T}$ satisfies condition (ii) from Definition 1. It is also proved in [13, Lemma 8] that the couple $\mathcal{E}:=\tilde{\mathcal{E}}_{\lambda_{n}}$ and $Q:=Q_{n-1, T}$ satisfies the same condition. To show that also other hypotheses of Theorem 3 are satisfied, we need some technical lemmas.

Lemma 4 If (6) is satisfied, then there exist $R>0$ and $\delta>0$ such that $\left\langle\mathcal{F}^{\prime}(s u), u\right\rangle \leq-\delta$ for any $s \geq R$ and $u \in \eta\left(1, \Lambda_{n}\right)$.

Proof We proceed via contradiction and assume that there exist $s_{k} \rightarrow \infty$ and $u_{k} \in \eta\left(1, \Lambda_{n}\right)$ such that

$$
\limsup _{k \rightarrow \infty}\left\langle\mathcal{F}^{\prime}\left(s_{k} u_{k}\right), u_{k}\right\rangle \geq 0
$$

Since $\eta\left(1, \Lambda_{n}\right)$ is compact, we may assume, without loss of generality, that $u_{k} \rightarrow u_{0}$ in $\eta\left(1, \Lambda_{n}\right)$ for some $u_{0} \in \eta\left(1, \Lambda_{n}\right)$.

If $u_{0} \neq \pm p^{\frac{1}{p}} \phi_{n}$, then there exists $\varepsilon>0$ such that

$$
\int_{0}^{1}\left|u_{0}^{\prime}(x)\right|^{p} \mathrm{~d} x-\lambda_{n} \int_{0}^{1}\left|u_{0}(x)\right|^{p} \mathrm{~d} x \leq-\varepsilon
$$

Hence, there exists $k_{\varepsilon} \in \mathbb{N}$ such that for any $k \geq k_{\varepsilon}$ we have

$$
\int_{0}^{1}\left|u_{k}^{\prime}(x)\right|^{p} \mathrm{~d} x-\lambda_{n} \int_{0}^{1}\left|u_{k}(x)\right|^{p} \mathrm{~d} x \leq-\frac{\varepsilon}{2}
$$


This implies

$$
\left\langle\mathcal{F}^{\prime}\left(s_{k} u_{k}\right), u_{k}\right\rangle \leq-\frac{\varepsilon}{2} s_{k}^{p-1}+\sum_{j=1}^{r} I_{j}\left(s_{k} u_{k}\left(t_{j}\right)\right) u_{k}\left(t_{j}\right)-\int_{0}^{1} f(x) u_{k}(x) \mathrm{d} x
$$

for $k \geq k_{\varepsilon}$. However, this contradicts (17).

$$
\text { If } u_{0}=p^{\frac{1}{p}} \phi_{n} \text {, we still have }
$$

$$
\int_{0}^{1}\left|u_{k}^{\prime}(x)\right|^{p} \mathrm{~d} x-\lambda_{n} \int_{0}^{1}\left|u_{k}(x)\right|^{p} \mathrm{~d} x \leq 0
$$

and so

$$
\left\langle\mathcal{F}^{\prime}\left(s_{k} u_{k}\right), u_{k}\right\rangle \leq \sum_{j=1}^{r} I_{j}\left(s_{k} u_{k}\left(t_{j}\right)\right) u_{k}\left(t_{j}\right)-\int_{0}^{1} f(x) u_{k}(x) \mathrm{d} x
$$

for all $k \in \mathbb{N}$. The boundedness of $I_{j}, j=1,2, \ldots, r$, and uniform convergence $u_{k} \rightarrow p^{\frac{1}{p}} \phi_{n}$ as $k \rightarrow \infty$ (due to continuous embedding $X \hookrightarrow C[0,1]$ ) then yield

$$
\begin{aligned}
\lim _{k \rightarrow \infty}\left\langle\mathcal{F}^{\prime}\left(s_{k} u_{k}\right), u_{k}\right\rangle & \leq p^{\frac{1}{p}}\left(\sum_{i=1}^{r_{+}} I_{i}^{\tau}(+\infty) \phi_{n}\left(\tau_{i}\right)+\sum_{j=1}^{r_{-}} I_{j}^{\sigma}(-\infty) \phi_{n}\left(\sigma_{j}\right)-\int_{0}^{1} f(x) \phi_{n}(x) \mathrm{d} x\right) \\
& <0
\end{aligned}
$$

by the first inequality in (6). This contradicts (17) again. Notice that by the boundedness of $I_{j}$ we have

$$
\left(p \hat{I}_{j}\left(u_{k}\left(t_{j}\right)\right)-I_{j}\left(u_{k}\left(t_{j}\right)\right)\right) v_{k}\left(t_{j}\right) \rightarrow 0 \quad \text { as } k \rightarrow \infty
$$

if $t_{j}$ is a zero point of $\phi_{n}$ for some $j \in\{1,2, \ldots, r\}$. The case $u_{0}=-p^{\frac{1}{p}} \phi_{n}$ is proved similarly using the second inequality in (6).

Lemma 5 If (6) is satisfied, then there exists $T>0$ such that

$$
\inf _{u \in \mathcal{E}_{\lambda_{n+1}}} \mathcal{F}(u)>\sup _{u \in \partial \tilde{Q}_{n, T}} \mathcal{F}(u)
$$

Proof There exists $\alpha \in \mathbb{R}$ such that for any $u \in \mathcal{E}_{\lambda_{n+1}}$ we have

$$
\mathcal{F}(u) \geq \frac{1}{p}\left(\lambda_{n+1}-\lambda_{n}\right)\|u\|_{L^{p}(0,1)}^{p}+\sum_{j=1}^{r} \int_{0}^{u\left(t_{j}\right)} I_{j}(\zeta) \mathrm{d} \zeta-\int_{0}^{1} f(x) u(x) \mathrm{d} x>\alpha .
$$

By Lemma 4 there exists $c \in \mathbb{R}$ such that for all $s>R$ and $u \in \eta\left(1, \Lambda_{n}\right)$ we have

$$
\mathcal{F}(s u)=\mathcal{F}(R u)+\mathcal{F}(s u)-\mathcal{F}(R u)=\mathcal{F}(R u)+\int_{R}^{s}\left\langle\mathcal{F}^{\prime}(\zeta u), u\right\rangle \mathrm{d} \zeta \leq c-\delta(s-R) .
$$


Thus there exists $T>R$ such that

$$
\mathcal{F}(s u) \leq c-\delta(s-R)<\alpha
$$

for all $s \geq T, u \in \eta\left(1, \Lambda_{n}\right)$. In particular, $\mathcal{F}(u)<\alpha$ for all $u \in \partial \tilde{Q}_{n, T}$ and (18) is proved.

Now we can finish the proof of Theorem 2 under assumption (6). Indeed, it follows from (18) that $\mathcal{E}_{\lambda_{n+1}} \cap \partial \tilde{Q}_{n, T}=\emptyset$ and thus the hypotheses of Theorem 3 hold with $\mathcal{E}:=\mathcal{E}_{\lambda_{n+1}}$ and $Q:=\tilde{Q}_{n, T}$. It then follows that $\mathcal{F}$ has a critical point and hence (1), (2) has a solution.

Next we show that the sets $\mathcal{E}:=\tilde{\mathcal{E}}_{\lambda_{n}}$ and $Q:=Q_{n-1, T}$ satisfy the hypotheses of Theorem 3 if (5) is satisfied.

The principal difference consists in the fact that, in contrast with $\eta\left(1, \Lambda_{n}\right)$, the set $\eta\left(-1, \mathcal{E}_{\lambda_{n}} \cap \mathcal{S}\right)$ is not compact. That is why one more technical lemma is needed.

Lemma 6 For any $\varepsilon^{\prime}>0$, there exists $\delta>0$ such that

$$
E(u) \geq \lambda_{n}+\delta
$$

for $u \in \eta\left(-1, \mathcal{E}_{\lambda_{n}} \cap \mathcal{S}\right) \backslash B_{\varepsilon^{\prime}}\left( \pm \phi_{n}\right)$. (Here $B_{\varepsilon^{\prime}}\left( \pm \phi_{n}\right)$ is the ball in $X$ centered at $\pm \phi_{n}$ with radius $\varepsilon^{\prime}$.)

Proof We note that the pseudo-gradient flow $\eta$ from Lemma 3 is constructed as a solution of the initial value problem $\frac{d}{d t} \eta(t, u)=-\tilde{v}(\eta(t, u)), \eta(0, \cdot)=\mathrm{id}$, where

$$
\tilde{v}(u)= \begin{cases}\psi(u) \operatorname{dist}\left(u, \mathcal{K}_{\lambda_{n}}\right) v(u) & \text { for } u \in \tilde{\mathcal{S}}:=\left\{w \in \mathcal{S}: E^{\prime}(w) \neq 0\right\} \\ 0 & \text { for } u \in \mathcal{S} \backslash \tilde{\mathcal{S}}\end{cases}
$$

$v(u)$ is a locally Lipschitz continuous symmetric pseudo-gradient vector field associated with $E$ on $\tilde{\mathcal{S}}$ and $\psi \rightarrow[0,1]$ is a smooth function such that $\psi(u)=1$ for $u$ satisfying $\lambda_{n}-$ $\tilde{\varepsilon} \leq E(u) \leq \lambda_{n}+\tilde{\varepsilon}$ and $\psi(u)=0$ for $u$ satisfying $E(u) \leq \lambda_{n}-\varepsilon$ or $\lambda_{n}+\varepsilon \leq E(u)$.

Let $\varepsilon^{\prime}>0$ and $u \in \eta\left(-1, \mathcal{E}_{\lambda_{n}} \cap \mathcal{S}\right) \backslash B_{\varepsilon^{\prime}}\left( \pm \phi_{n}\right)$. Without loss of generality, we may assume that $E(u) \leq \lambda_{n}+\tilde{\varepsilon}$. Let $u_{0} \in \mathcal{E}_{\lambda_{n}} \cap \mathcal{S}$ be such that $u=\eta\left(-1, u_{0}\right)$. Observe that there is a constant $M>0$ such that for $t \in[-1,1]$ we have

$$
\left\|\frac{d}{d t} \eta\left(t, u_{0}\right)\right\| \leq\left\|\tilde{v}\left(\eta\left(t, u_{0}\right)\right)\right\| \leq \operatorname{dist}\left(\eta\left(t, u_{0}\right), \mathcal{K}_{\lambda_{n}}\right)\left\|\tilde{v}\left(\eta\left(t, u_{0}\right)\right)\right\|<M .
$$

Hence $\eta\left(t, u_{0}\right) \notin B_{\frac{\varepsilon^{\prime}}{2}}\left( \pm \phi_{n}\right)$ for $t \in\left[-1,-1+\frac{\varepsilon^{\prime}}{2 M}\right]$. Since $E$ satisfies the Palais-Smale condition on $\mathcal{S}$ (see [13, Lemma 2]), there exists $\rho>0$ such that $\left\|E^{\prime}(u)\right\|_{*} \geq \rho$ for all $u \in\left\{w \in \mathcal{S}: \lambda_{n} \leq\right.$ $\left.E(w) \leq \lambda_{n}+\tilde{\varepsilon}\right\} \backslash B_{\frac{\varepsilon^{\prime}}{2}}\left( \pm \phi_{n}\right)$. Then

$$
\begin{aligned}
\left\|\frac{d}{d t} E\left(\eta\left(t, u_{0}\right)\right)\right\| & =\left\|\left\langle E^{\prime}\left(\eta\left(t, u_{0}\right)\right), \frac{d}{d t} \eta\left(t, u_{0}\right)\right\rangle\right\| \\
& =\left\|\psi\left(\eta\left(t, u_{0}\right)\right) \operatorname{dist}\left(\eta\left(t, u_{0}\right), \mathcal{K}_{\lambda_{n}}\right)\left\langle E^{\prime}\left(\eta\left(t, u_{0}\right)\right), v\left(\eta\left(t, u_{0}\right)\right)\right\rangle\right\| \\
& \geq 1 \cdot \frac{\varepsilon^{\prime}}{2} \cdot \min \left\{\left\|E^{\prime}\left(\eta\left(t, u_{0}\right)\right)\right\|, 1\right\}\left\|E^{\prime}\left(\eta\left(t, u_{0}\right)\right)\right\| \geq \frac{\varepsilon^{\prime}}{2} \rho^{2}
\end{aligned}
$$


for all $t \in\left[-1,-1+\frac{\varepsilon^{\prime}}{2 M}\right]$. The last but one inequality holds due to the following property of $v(u)$ :

$$
\left\langle E^{\prime}(u), v(u)\right\rangle>\min \left\{\left\|E^{\prime}(u)\right\|, 1\right\}\left\|E^{\prime}(u)\right\|
$$

(see [14] and [2]). We also used the fact that $\psi\left(\eta\left(t, u_{0}\right)\right) \equiv 1$ for $t \in[-1,0]$. Hence

$$
\begin{aligned}
E(u) & =E\left(\eta\left(-1, u_{0}\right)\right)=E\left(\eta\left(-1+\frac{\varepsilon^{\prime}}{2 M}, u_{0}\right)\right)+\int_{-1+\frac{\varepsilon^{\prime}}{2 M}}^{-1} \frac{\mathrm{d}}{\mathrm{d} t} E\left(\eta\left(t, u_{0}\right)\right) \mathrm{d} t \\
& \geq E\left(\eta\left(-1+\frac{\varepsilon^{\prime}}{2 M}, u_{0}\right)\right)+\frac{\varepsilon^{\prime}}{2} \rho^{2} \cdot \frac{\varepsilon^{\prime}}{2 M} \geq \lambda_{n}+\delta
\end{aligned}
$$

with $\delta=\frac{\left(\varepsilon^{\prime} \rho\right)^{2}}{4 M}$.

The following lemma is a counterpart of Lemma 4 in the case of condition (5).

Lemma 7 If (5) is satisfied, then there exist $R>0$ and $\delta>0$ such that $\left\langle\mathcal{F}^{\prime}(s u), u\right\rangle \geq \delta$ for any $s \geq R$ and $u \in \eta\left(-1, \mathcal{E}_{\lambda_{n}} \cap \mathcal{S}\right)$.

Proof We proceed via contradiction and assume that there exist $s_{k} \rightarrow \infty$ and $u_{k} \in$ $\eta\left(-1, \mathcal{E}_{\lambda_{n}} \cap \mathcal{S}\right)$ such that

$$
\limsup _{k \rightarrow \infty}\left\langle\mathcal{F}^{\prime}\left(s_{k} u_{k}\right), u_{k}\right\rangle \leq 0
$$

If there is $\varepsilon^{\prime}>0$ such that $u_{k} \in \eta\left(-1, \mathcal{E}_{\lambda_{n}} \cap \mathcal{S}\right) \backslash B_{\varepsilon^{\prime}}\left( \pm \phi_{n}\right)$ for all $k$ large enough, then Lemma 6 leads to the estimate

$$
\left\langle\mathcal{F}^{\prime}\left(s_{k} u_{k}\right), u_{k}\right\rangle \geq \delta s_{k}^{p-1}+\sum_{j=1}^{r} I_{j}\left(s_{k} u_{k}\left(t_{j}\right)\right) u_{k}\left(t_{j}\right)-\int_{0}^{1} f(x) u_{k}(x) \mathrm{d} x
$$

contradicting (20). Thus it must be $u_{k} \rightarrow \pm p^{\frac{1}{p}} \phi_{n}$ as $k \rightarrow \infty$. If $u_{k} \rightarrow p^{\frac{1}{p}} \phi_{n}$ as $k \rightarrow \infty$, we still have

$$
\int_{0}^{1}\left|u_{k}^{\prime}(x)\right|^{p} \mathrm{~d} x-\lambda_{n} \int_{0}^{1}\left|u_{k}(x)\right|^{p} \mathrm{~d} x \geq 0
$$

and so

$$
\left\langle\mathcal{F}^{\prime}\left(s_{k} u_{k}\right), u_{k}\right\rangle \geq \sum_{j=1}^{r} I_{j}\left(s_{k} u_{k}\left(t_{j}\right)\right) u_{k}\left(t_{j}\right)-\int_{0}^{1} f(x) u_{k}(x) \mathrm{d} x
$$

for all $k \in \mathbb{N}$. Similar arguments as in the proof of Lemma 4 lead to

$$
\begin{aligned}
\lim _{k \rightarrow \infty}\left\langle\mathcal{F}^{\prime}\left(s_{k} u_{k}\right), u_{k}\right\rangle & \geq p^{\frac{1}{p}}\left(\sum_{i=1}^{r_{+}} I_{i}^{\tau}(+\infty) \phi_{n}\left(\tau_{i}\right)+\sum_{j=1}^{r_{-}} I_{j}^{\sigma}(-\infty) \phi_{n}\left(\sigma_{j}\right)-\int_{0}^{1} f(x) \phi_{n}(x) \mathrm{d} x\right) \\
& >0
\end{aligned}
$$


by the second inequality in (5). This contradicts (20) again. The case $u_{k} \rightarrow-p^{\frac{1}{p}} \phi_{n}$ as $k \rightarrow$ $\infty$ is proved similarly but using the first inequality in (5).

Lemma 8 If (5) is satisfied, then there exists $T>0$ such that

$$
\inf _{u \in \tilde{\mathcal{E}}_{\lambda_{n}}} \mathcal{F}(u)>\sup _{u \in \partial Q_{n-1, T}} \mathcal{F}(u)
$$

Proof By Lemma 7 there exists $d \in \mathbb{R}$ such that for all $s>R$ and $u \in \eta\left(-1, \mathcal{E}_{\lambda_{n}} \cap \mathcal{S}\right)$ we have

$$
\mathcal{F}(s u)=\mathcal{F}(R u)+\mathcal{F}(s u)-\mathcal{F}(R u)=\mathcal{F}(R u)+\int_{R}^{s}\left\langle\mathcal{F}^{\prime}(\zeta u), u\right\rangle \mathrm{d} \zeta \geq d+\delta(s-R) .
$$

Hence, there exists $\alpha \in \mathbb{R}$ such that for any $u \in \tilde{\mathcal{E}}_{\lambda_{n}}$ we have

$$
\mathcal{F}(u)>\alpha
$$

On the other hand, for any $s>0$ and $u \in \Lambda_{n-1}$, we get

$$
\begin{aligned}
\mathcal{F}(s u) & =\frac{1}{p}\left(\lambda_{n-1}-\lambda_{n}\right)\|s u\|_{L^{p}(0,1)}^{p}+\sum_{j=1}^{r} \int_{0}^{s u\left(t_{j}\right)} I_{j}(\zeta) \mathrm{d} \zeta-s \int_{0}^{1} f(x) u(x) \mathrm{d} x \\
& =\left(\lambda_{n-1}-\lambda_{n}\right) s^{p}+\sum_{j=1}^{r} \int_{0}^{s u\left(t_{j}\right)} I_{j}(\zeta) \mathrm{d} \zeta-s \int_{0}^{1} f(x) u(x) \mathrm{d} x .
\end{aligned}
$$

Thus, there exists $T>0$ such that, for $u \in \partial Q_{n-1, T}$,

$$
\mathcal{F}(u)<\alpha
$$

and (21) is proved.

It follows that the sets $\mathcal{E}:=\tilde{\mathcal{E}}_{\lambda_{n}}$ and $Q:=Q_{n-1, T}$ satisfy the hypotheses of Theorem 3 if (5) is satisfied. The proof of Theorem 2 is thus completed.

Final remark Reviewers of our manuscript suggested to include some recent references on impulsive problems. Variational approach to impulsive problems can be found, e.g., in [17-21]. The last reference deals with the $p$-Laplacian with the variable exponent $p=p(t)$. Singular impulsive problems are treated in [22-24]. Impulsive problems are still 'hot topic' attracting the attention of many mathematicians and the bibliography on that topic is vast.

Competing interests

The authors declare that they have no competing interests.

Authors' contributions

Both authors read and approved the final manuscript.

Author details

${ }^{1}$ Department of Mathematics, University of West Bohemia, Univerzitní 22, Plzeň, 306 14, Czech Republic. ${ }^{2}$ NTIS, University of West Bohemia, Univerzitní 22, Plzeň, 306 14, Czech Republic. 


\section{Acknowledgements}

This research was supported by Grant 13-00863S of the Grant Agency of Czech Republic and by the European Regional Development Fund (ERDF), project 'NTIS - New Technologies for the Information Society', European Centre of Excellence, CZ.1.05/1.1.00/02.0090

\section{Received: 5 December 2013 Accepted: 7 March 2014 Published: 24 Mar 2014}

\section{References}

1. Drábek, P, Langerová, M: On the second order equations with nonlinear impulses - Fredholm alternative type results. Topol. Methods Nonlinear Anal. (to appear)

2. Ghoussoub, N: Duality and Perturbation Methods in Critical Point Theory. Cambridge University Press, Cambridge (1993)

3. del Pino, M, Drábek, P, Manásevich, R: The Fredholm alternative at the first eigenvalue for the one dimensional p-Laplacian. J. Differ. Equ. 151, 386-419 (1999)

4. Drábek, P, Manásevich, R: On the closed solutions to some nonhomogeneous eigenvalue problems with p-Laplacian. Differ. Integral Equ. 12, 773-788 (1999)

5. Lindqvist, P: Some remarkable sine and cosine functions. Ric. Mat. XLIV, 269-290 (1995)

6. del Pino, M, Elgueta, M, Manásevich, R: A homotopic deformation along $p$ of a Leray-Schauder degree result and existence for $\left(\left|u^{\prime}\right|^{p-2} u^{\prime}\right)^{\prime}+f(t, u)=0, u(0)=u(T), p>1$. J. Differ. Equ. 80, 1-13 (1989)

7. Fleckinger, J, Hernández, J, Takáč, P, de Thélin, F: Uniqueness and positivity for solutions of equations with the p-Laplacian. In: Caristi, G, Mitidieri, E (eds.) Proceedings of the Conference on Reaction-Diffusion Equations, Trieste, Italy. Lecture Notes in Pure and Applied Math., vol. 194, pp. 141-155 (1995)

8. Nieto, JJ, O'Regan, D: Variational approach to impulsive differential equations. Nonlinear Anal., Real World Appl. 10, 680-690 (2009)

9. Drábek, P, Girg, P, Takáč, P, Ulm, M: The Fredholm alternative for the $p$-Laplacian: bifurcation from infinity, existence and multiplicity. Indiana Univ. Math. J. 53, 433-482 (2004)

10. Manásevich, R, Takáč, P: On the Fredholm alternative for the $p$-Laplacian in one dimension. Proc. Lond. Math. Soc. 84, 324-342 (2002)

11. Drábek, P, Milota, J: Methods of Nonlinear Analysis, Applications to Differential Equations, 2nd edn. Springer, Basel (2013)

12. Drábek, P: Solvability and Bifurcations of Nonlinear Equations. Pitman Res. Notes in Math. Series, vol. 264. Longman, Harlow (1992)

13. Drábek, P, Robinson, SB: Resonance problems for the one-dimensional p-Laplacian. Proc. Am. Math. Soc. 128, 755-765 (1999)

14. Struwe, M: Variational Methods; Applications to Nonlinear Partial Differential Equations and Hamiltonian Systems. Springer, New York (1990)

15. Drábek, P, Robinson, SB: Resonance problems for the $p$-Laplacian. J. Funct. Anal. 169, 189-200 (1999)

16. Drábek, P, Robinson, SB: On the generalization of the Courant nodal domain theorem. J. Differ. Equ. 181, 58-71 (2001)

17. Chen, $\mathrm{H}, \mathrm{He}, \mathrm{Z}$ : Variational approach to some damped Dirichlet problems with impulses. Math. Methods Appl. Sci. 36(18), 2564-2575 (2013)

18. Otero-Espinar, V, Pernas-Castaño, T: Variational approach to second-order impulsive dynamic equations on time scales. Bound. Value Probl. 2013, 119 (2013)

19. Xiao, J, Nieto, JJ: Variational approach to some damped Dirichlet nonlinear impulsive differential equations. J. Franklin Inst. 348(2), 369-377 (2011)

20. Galewski, M: On variational impulsive boundary value problems. Cent. Eur. J. Math. 10(6), 1969-1980 (2012)

21. Galewski, M, O’Regan, D: Impulsive boundary value problems for $p(t)$-Laplacian's via critical point theory. Czechoslov. Math. J. 62(4), 951-967 (2012)

22. Sun, J, Chu, J, Chen, H: Periodic solution generated by impulses for singular differential equations. J. Math. Anal. Appl. 404(2), 562-569 (2013)

23. Chu, J, Nieto, JJ: Impulsive periodic solutions of first-order singular differential equations. Bull. Lond. Math. Soc. 40(1), 143-150 (2008)

24. Sun, J, O'Regan, D: Impulsive periodic solutions for singular problems via variational methods. Bull. Aust. Math. Soc. 86(2), 193-204 (2012)

10.1186/1687-2770-2014-64

Cite this article as: Drábek and Langerová: Quasilinear boundary value problem with impulses: variational approach to resonance problem. Boundary Value Problems 2014, 2014:64 